\title{
Endodontic treatment failure caused by arsen utilization as the devitalization material
}

\author{
Endang Sukartini, Kurniasri Darliana
}

Department of Conservative Dentistry Faculty of Dentistry Universitas Padjadjaran

\begin{abstract}
Root canal treatment diagnose as puplitis irreversible can be treated in a non vital condition with devitalization material. Arsenic is one of devitalization material that used in inflammatory pulp tissue before it is going to endodontic treatment. The long term use of it or the leak of using this arsenic can cause the toxic effect of the pulp tissue. The case is going to report is about the damage of gingival tissue and alveolar that caused by the uncarefullness using of arsenic. Unappropriate arsenic applications cause the leak that able to spread to gingival tissue and will become necrosis. Now a day, the using of arsenic begin to leave because the toxicity sad effect. This report is going to report how much the damage using arsenic trioxide $\left(\mathrm{As}_{2} \mathrm{O}_{3}\right)$ and the effort from the leakness.
\end{abstract}

Key words: Failure of endodontic treatment, devitalization, arsenic

\section{INTRODUCTION}

Endodontic treatment is often performed either in vital, non vital or devital teeth. Devitalization treatment can be done to teeth with irreversible pulpitis using pulp devitalization material including arsen trioxide $\left(\mathrm{As}_{2} \mathrm{O}_{3}\right)$ or euparal.

Arsen as a pulp devitalization material is easy to handle. However, in a long term usage (more than two days), arsen may create unwanted damages in the tissue. This is caused by a very toxic and non self limiting nature of arsen. By referring to the danger that may happen, the devitalization using arsen needs a very careful management. The placement of arsen in the cavity should be precise especially when we deal with a wide cavity or class II cavity that often enable arsen to leak through the filler that it can reach gingival papillae and even alveolar process. This condition may lead to gingival and alveolar process necrosis. In this case, the endodontic treatment will lead to failure. Arsen-induced osteomyelitis can be treated using combination of pharmacotherapy and surgery.

The use of arsen is now neglected due to the toxic stimulation effect for tissue and as the substitution, euparal is used. This paper reports the presence of necrosis and osteomyelitis as well as sinusitis due to the connection of sinus and oral cavity in alveolar process caused by arsen use and the efforts needed to prevent the leak. Osteomyelitis case that is caused by arsen use as an inflammed pulp tissue devitalization material has often happened.

This paper describes a jaw necroses caused by arsen in left and right maxilla due to the arsen leak to the periapical tissue and alveolar bone 
right to the pulp that is treated endodontically. This condition has created bilateral oroanthral fistula.

\section{CASE REPORT}

A 33 female patient came with a complaint of uneasiness and hot feeling in the area of left upper molar tooth (in 2008). The patient was treated more or less 3 months before by a dentist at the company where she works. According to her dentist, the treatment is already finished and an amalgam filling has been made. The patient complained that when she bent her body and when she bowed in kneeling position for praying she felt a pain under her eyes, especially in the left jaw area. The area was also painful if it was pressed.

The patient came to a dentist private practice with a good general condition with a general status in a normal limit. There is no lymph node enlargement on the neck. The pressure pain was felt in the left infra orbital area.

From the intraoral examination, it was revealed that tooth 16 and 26 were already missing, there was a gap in the mesial part of tooth 26 . The gingival necrosis was already wide with a deep alveolar bone necrosis and caries in the cervical area of the distal part of tooth 27.

The supporting examination used was $3 \mathrm{D} \mathrm{X}$ ray photo with a result that showed left and right acute trauma. In the left region, pus was filling the sinus cavity. The sinus border seemed interrupted in the apical area of tooth 17 and 27.

The patient was referred to an ENT doctor for sinusitis drainage and after the ENT stated that the sinusitis was resolved and there was no pressure pain anymore in the infraorbital area, the patient was referred to the oral surgery department for an extraction.

\section{DISCUSSION}

Arsen is the most active toxic material that is often used for pulp devitalization material. However, nowadays the use of this agent is neglected because it can kill the pulp, cause periodontal membrane damage, dentoalveolar membrane inflammation, abscess from osteomyelitis and even death.
In the $19^{\text {th }}$ decade, arsen was still used by several dentists to devitalize dental pulp with narrow and swollen canal or for patient with a severe systemic and local anesthetic allergy. However, tissue damage is rarely found among patients.

Smart and Barnes in 1991 reported a case where arsen containing copper was used to treat upper first molar leading to bone necrosis and dental loss.

In general, arsen is used for devitalizing inflamed pulp tissue after a root canal treatment. Application that is more than 3 days or the leak of this agent may cause toxic effects on the pulp tissues and a necrosis in periodontal tissue and alveolar bone.

Osteomyelitis due to arsen leak often happens. Therefore, practitioners should be careful in using arsen since it can diffuse into the periodontal tissue through the apical canal, lateral canal, accessory canal through a perforation and leaking restoration. The treatment for this case is sequesterectomy and non vital alveolar bone excision.

The work mechanism of arsen is making smooth muscle of the capillaries and blood vessels paralyze so that a hyperemic vasodilatation and exudation may happen. Blood vessel wall damage may also happen that leads to hemorrhage and thrombosis leading to non functioning blood vessel and nutrition disorder that ends up in necrotic pulp.

Arsen application into the cavity needs a careful manipulation because it is easy for leaking towards the gingival papillae and alveolar process to happen. The leak of arsen maybe caused by the followings: (1) Unhermetic covering of temporary filling that enable arsen to escape through the filler and penetrate the gingiva. (2) In class II cavity or hidden caries condition. (3) Saliva can enter the cavity and bring the arsen out of the cavity. (4) The presence of lateral root canal or accessory root canal that enable arsen to reach the periodontal tissue. (5) The presence of perforation at the bifurcation. (6) Long term use of arsen, more than 1-2 days.

Due to the danger that may happen, some efforts are needed to prevent the leak of arsen, including: (1) Using a more adhesive temporary filler. (2) Pre-blocking the proximal part of the 
tooth before applying arsen by making an artificial wall to prevent arsen leak through the temporary filler. To create an artificial wall, glass ionomer cement can be used because it has adhesive property for dental tissue that it is expected that a leak will not happen. (3) Making x-ray photo before applying arsen to see whether there is any hidden caries, lateral or accessory root canal and

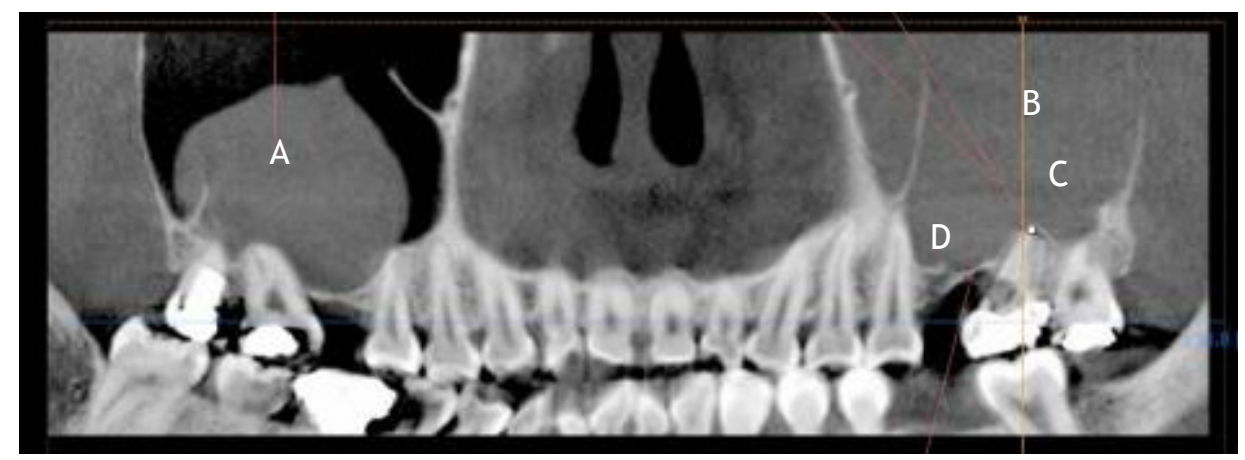

Figure 1. 3-D Radiographic of tooth 27.

A. Inflamation tissue of sinus maxillaris dextra (suspect: sinusitis maxillaris dextra), B. Inflamation tissue of sinus maxillaris sinistra (suspect: sinusitis maxillaris sinistra), C. Diffuse radiolucent lesions at periapical tooth 27 ,

D. Invagination of inferior wall sinus maxillaris sinistra due to arsenic perforation that makes sinus wall perforation.

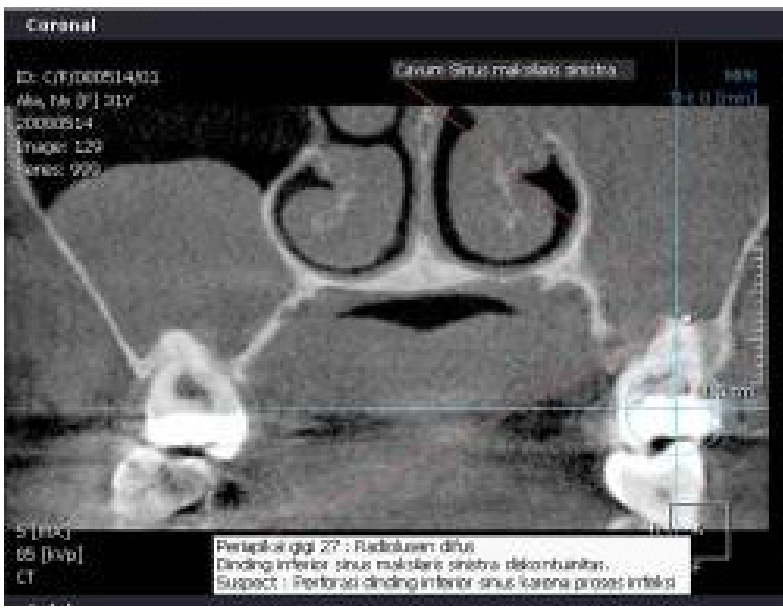

Figure 2. Frontal view

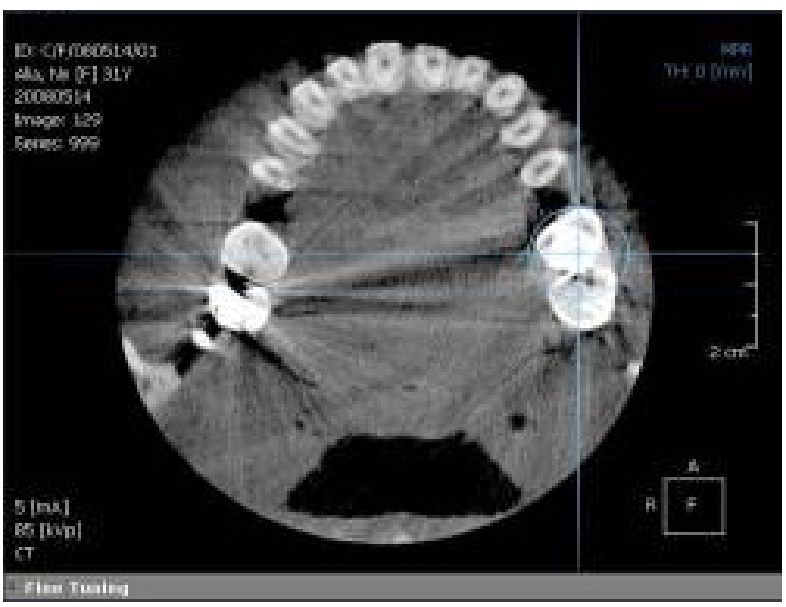

Figure 4. Occlusal view

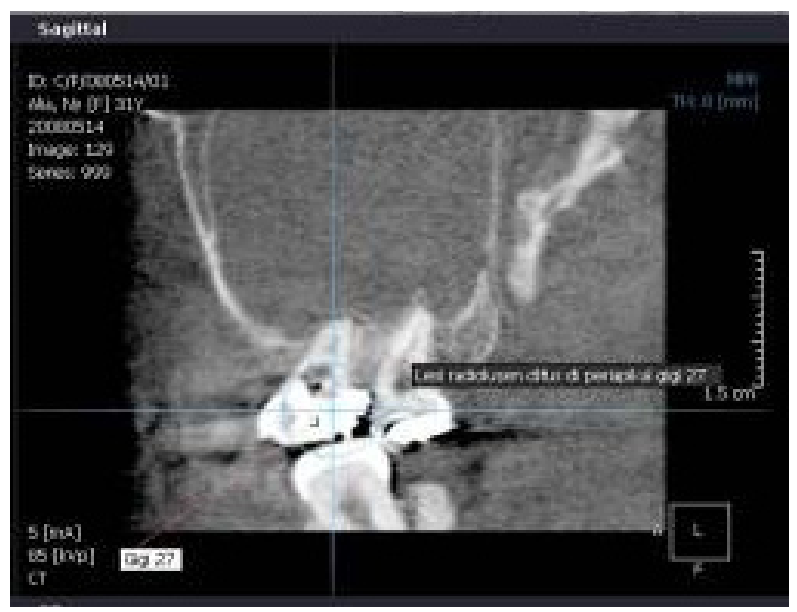

Figure 3. Sagital view

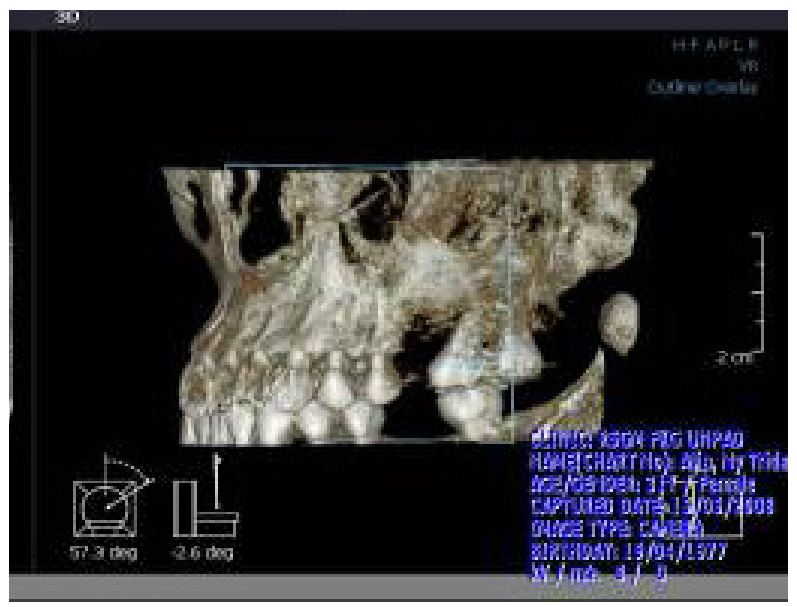

Figure 5. Perforation at sinus wall due to arsenic leakage. 
perforation at the furcation. By examining the $x$ ray photo we will know where to put the arsen properly by removing any existing caries and filling any existing perforation first. (4) Assuring the patient to come back in two day time considering the toxic nature of arsen and the strength of the temporary filler used.

\section{CONCLUSION}

Even though the operator has filled the cavity using a temporary filler in an appropriate manner, a leak may still happen because saliva can enter the cavity and bring arsen outside the cavity. Arsen in the paste form is often able to enter the periodontal tissue through the main, lateral or accessory root canal or even through any perforation at the furcation. By considering the danger that may happen, arsen as the devitalization material is not used anymore.

\section{REFERENCES}

1. Dumlu A, Hata $M$, Shiono $M$, Orime $Y$. Osteomyelitis due to arsenic trioxide use for tooth devitalization. Int Endodontic J 2007;40(4):317-22.

2. Garip H, Goldberg F, Massone E, Artaza L. Management of arsenic trioxide necrosis in maxilla. J Endodontics 2003;30(10):732-6.

3. Hyson JM. A history of arsenic in dentistry. J Calif Dent Assoc 2007:35(2);135-9.

4. Matthew JD. Pink teeth resulting from endodontic therapy. J Am Dent Assoc. 2007;131(11):1598-9.

5. Tanu I. Farmakologi dan terapi. $4^{\text {th }}$ ed. Jakarta: Fakultas Kedokteran Universitas Indonesia.

6. Yalgin B, Aybar B, Haznedaroglu F, Yucel MD. Bilateral oroantral fistulas following devitalization of teeth by arsenic trioxide: A Case Report. J Endodontics 2003;29(3):205-7. 